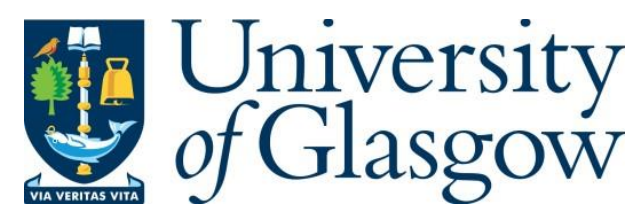

Krauss, R., Brante, G., Rayel, O. K., Demo Souza, R., Onireti, O. and Imran, M. A. (2018) On the Area Energy Efficiency of Multiple Transmit Antenna Small Base Stations. In: IEEE GLOBECOM, Singapore, 4-8 Dec 2017, ISBN 9781509050192.

There may be differences between this version and the published version. You are advised to consult the publisher's version if you wish to cite from it.

http://eprints.gla.ac.uk/144920/

Deposited on: 27 July 2017

Enlighten - Research publications by members of the University of Glasgow http://eprints.gla.ac.uk 


\title{
On the Area Energy Efficiency of Multiple Transmit Antenna Small Base Stations
}

\author{
Roberto Krauss*, Glauber Brante*, Ohara Kerusauskas Rayel*, Richard Demo Souza ${ }^{\dagger}$, \\ Oluwakayode Onireti ${ }^{\ddagger}$, Muhammad Ali Imran ${ }^{\ddagger}$ \\ *Federal University of Technology - Paraná, UTFPR, Curitiba, Brazil \\ E-mails: roberto.krauss.eng@gmail.com, \{gbrante, oharakr\}@utfpr.edu.br \\ ${ }^{\dagger}$ Federal University of Santa Catarina, UFSC, Florianópolis, Brazil \\ E-mail: richard.demo@ufsc.br \\ $\ddagger$ University of Glasgow, Glasgow, UK \\ E-mails: \{oluwakayode.onireti, muhammad.imran\}@glasgow.ac.uk
}

\begin{abstract}
We analyze the area energy efficiency (AEE) of spatial multiplexing (SM) and transmit antenna selection (TAS), considering a realistic power consumption model for small base stations (BSs), which includes the power consumed by the backhaul as well as different interference attenuation levels. Our results show an optimum number of BSs for each technique that maximizes the AEE. Moreover, we also show that TAS has a larger AEE than SM when the demand for system capacity is low, while SM becomes more energy efficient when the demanded capacity is larger. Additionally, when the capacity demand and the area to be covered are fixed, the number of BSs needed to be deployed is smaller for SM than for the other techniques. Finally, the system performance in terms of AEE is shown to be strongly dependent on the amount of interference, which in turn depends on the employed interference-mitigation scheme, and on the employed power consumption model.
\end{abstract}

\section{INTRODUCTION}

By 2020, the aggregate traffic is expected to be between 8 and 12 times greater than today [1], [2]. Such growing demand requires more base stations (BSs), which in turn may increase the network power consumption. An important task in network planning is finding the optimal number of BSs that achieves a desired quality of service while maximizing the energy efficiency. Moreover, it is well known that choosing a proper antenna technique can lead to either meeting the increased traffic demand, or reducing the power consumption [3]. Looking forward to improve spectral efficiency, the long term evolution (LTE) cellular network 4G standard [4] employs multiple antenna (MIMO) technologies in order to mitigate the effects of fading, providing diversity gains, or to increase the network capacity, providing multiplexing gains through spatial multiplexing (SM) techniques. However, these techniques also lead to a greater energy consumption as a result of the multiple radio frequency (RF) chains, specially due to the power amplifier consumption that corresponds to $55-60 \%$ of the total consumption in a BS [5].

Another MIMO technique that has been already considered for energy efficient deployments is the transmit antenna selection (TAS), in which only one RF chain remains active at the transmitter [6]. It is worth noting that LTE already employs TAS, but at the user equipment (UE) only [7], while at the BS side it could lead to greater area energy efficiency (AEE), with the same diversity order as in MIMO [8]. Moreover, according to [3], when analyzed through a realistic power consumption (PCM) model, TAS is more energy efficient when compared to $\mathrm{SM}$ in the low to medium spectral efficiency region.

The use of a realistic PCM is important to evaluate the energy efficiency, as shown, e.g., in [3], [9]-[11], which take into account several components that consume power in a BS, such as the AC-DC main power unit, cooling and DC-DC power supplies, as well as the RF power amplifier chain for communications. Additionally, the works in [12], [13] have shown that the power consumed by the backhaul - i.e., the power consumed by the aggregation switches, which is a function of the network traffic - should not be neglected into a complete network energy efficiency evaluation, since it may be the bottleneck in terms of energy consumption.

Severe inter-cell interference may arise due to dense network deployments. This problem was first addressed in 3GPP LTE standard release 8 [14], where the inter-cell interference coordination (ICIC) was introduced to allocate different frequency resources to the UEs at the cell edge. Since then, the releases 9 and 10 employ an enhanced ICIC [14], allocating different subframes between macro and small cells, also enhancing intra-cell interference. Release 11 introduces coordinated multi-point transmit and reception (CoMP) [15], where a dynamic coordination for transmission and reception of signals at multiple cells is made. CoMP is a cooperation technology where one or more BSs serve the UE in order to mitigate interferences and to achieve better throughputs. In [16], the combination of fractional frequency reuse and the cooperative/coordination of multiple separated cells, achieve an improvement for signal-to-interference-plus-noise ratio (SINR) at the cell edge of approximately $13 \mathrm{~dB}$. Nevertheless, channel state information (CSI) must be constantly shared between UEs and BSs in order to make scheduling possible, which due to imperfections in channel estimation and number of served UEs may lead to different levels of interference cancellation.

In this paper, we analyze the energy efficiency of SM and TAS at the cell edge in a $4 \mathrm{G}$ cellular network consisting of small BSs. In this scenario the UE is subjected to in- 
terference from other neighbor small BSs. We also assume that interference may not be fully canceled due to, e.g., the interference mitigation technique or imperfect CSI estimation, so that we consider a fraction of residual interference denoted by $\kappa$. Moreover, we employ a realistic PCM that combines [3] and [13], i.e., it scales with the number of active antennas at the BS for the different MIMO techniques [3], at the same time that it includes the backhaul power consumption [13]. Differently from [9]-[11], by considering TAS we observe different trade-offs in terms of AEE between the MIMO techniques. For instance, TAS stands out with the largest AEE when the demand for system capacity is low, while SM becomes more energy efficient when the capacity demand is larger. However, the point of the demanded system capacity that dictates the intersection between TAS and SM considerably changes depending on the employed PCM, e.g., if the backhaul or the fraction that scales with the number of antennas are considered or not. Moreover, we observe an optimum number of BSs that maximizes the AEE for each technique. By fixing the capacity demand and the area to be covered, TAS is more energy efficient but requires more BSs than SM. Finally, the performance in terms of AEE is shown to be strongly dependent on $\kappa$, so that conclusions in terms of the MIMO scheme that achieves the largest AEE may change with the performance of the interference mitigation technique in use.

\section{SYSTEM MODEL}

Let us consider a cellular network composed by hexagonal cells of radius $R$, covering an area $A$, in $\mathrm{km}^{2}$, so that the number of small base stations required for such area is $N_{\mathrm{BS}}=$ $\frac{2 A}{3 \sqrt{3} R^{2}}$. In the downlink direction, the signal transmitted by the $\mathrm{BS}$ and received by the $\mathrm{UE}$ is given by

$$
\mathbf{y}=\sqrt{\frac{P_{\mathrm{L}} P_{\mathrm{tx}}}{\widehat{m}_{\mathrm{t}}}} \mathbf{H} \mathbf{x}+\mathbf{w},
$$

where $P_{\mathrm{L}}=\frac{G \lambda^{2}}{L(4 \pi)^{2} d^{\alpha}}$ is the path loss, where $d$ is the transmission distance, $G$ is the antenna gain, $L$ is the link margin, $\lambda$ is the wavelength and $\alpha$ is the path loss exponent, $P_{\mathrm{tx}}$ is the transmit power of the BS, $\mathbf{H} \in \mathbb{C}^{m_{\mathrm{r}} \times \widehat{m}_{\mathrm{t}}}$ is the channel matrix composed by the fading coefficients $h_{i, j}$, where $m_{\mathrm{t}}$ is the number of transmit antennas, $\widehat{m}_{\mathrm{t}}$ is the number of active transmit antennas ${ }^{1}, m_{\mathrm{r}}$ is the number of receiving antennas, $\mathbf{x} \in \mathbb{C}^{\widehat{m}_{\mathrm{t}} \times 1}$ is the unit energy transmitted symbol vector, $\mathbf{y} \in \mathbb{C}^{m_{\mathrm{r}} \times 1}$ is the received symbol vector and $\mathbf{w} \in \mathbb{C}^{m_{\mathrm{r}} \times 1}$ is the zero-mean additive white Gaussian noise with variance $N_{0} / 2$ per dimension, where $N_{0}$ is the thermal noise power spectral density per Hertz. Then, the average signal-to-noise ratio (SNR) per receive antenna is written as

$$
\bar{\gamma}=\frac{P_{\mathrm{L}} P_{\mathrm{tx}}}{N_{0} W}
$$

where $W$ is the channel bandwidth.

Moreover, we also consider that the communication links are subjected to interference, which may not be fully canceled

\footnotetext{
${ }^{1}$ Notice that $\widehat{m}_{\mathrm{t}} \leq m_{\mathrm{t}}$, while the active antennas are selected according to the employed MIMO transmission scheme.
}

depending on the employed interference mitigation scheme, so that in our model we include a factor denoted by $\kappa \in[0,1]$ that multiplies the maximum interference power $P_{\mathrm{I}}$. Thus, the signal-to-interference power ratio (SIR) in the case of hexagonal cells becomes [17]

$$
\zeta=\frac{P_{\mathrm{L}} P_{\mathrm{tx}}}{\kappa P_{\mathrm{I}}}=\frac{3^{\frac{\alpha}{2}}}{6 \kappa},
$$

in which $\kappa=0$ yields $\zeta \rightarrow \infty$, i.e., full interference cancellation, while $\kappa=1$ considers the worst-case scenario with no interference cancellation at all.

Then, the average SINR for the UE at the cell edge is [17]

$$
\Gamma=\frac{P_{\mathrm{L}} P_{\mathrm{tx}}}{N_{0} W+\kappa P_{\mathrm{I}}}=\frac{\bar{\gamma}}{1+\bar{\gamma} \zeta^{-1}} .
$$

\section{A. Network Total Power}

To compute the network total power consumption, $P_{\text {net }}$, we employ a PCM combining [3] and [13], which also takes into account the number of active antennas at the BS. Thus,

$$
\left.P_{\text {net }}=N_{\mathrm{BS}}\left[\widehat{m}_{\mathrm{t}}\left(P_{0} P_{\mathrm{tx}}+P_{1}\right)+P_{2}\right)\right]+P_{\mathrm{bh}},
$$

where $P_{0}$ is a constant that encompasses the effects of the power amplifier drain efficiency, cooling, power supply and battery backup losses, $P_{1}$ represents the part of the circuitry power consumption that grows linearly with $\widehat{m}_{\mathrm{t}}$, while $P_{2}$ is the power consumption that does not depend on $\widehat{m}_{\mathrm{t}}$ [3], [9]. Moreover, $P_{\mathrm{bh}}$ is the power consumption of the backhaul ${ }^{2}$.

The power consumed by the backhaul takes into account the power consumed by the downlink interfaces $\left(P_{\mathrm{dl}}\right)$, uplink interfaces $\left(P_{\mathrm{ul}}\right)$ and the power consumed by the access switch $\left(P_{\mathrm{s}}\right)$, being written as [13]

$$
P_{\mathrm{bh}}=\left\lceil\frac{N_{\mathrm{BS}}}{\max _{\mathrm{dl}}}\right\rceil P_{\mathrm{s}}+N_{\mathrm{BS}} P_{\mathrm{dl}}+N_{\mathrm{ul}} P_{\mathrm{ul}},
$$

where $\lceil$.$\rceil is the ceil operation, \max _{\mathrm{dl}}$ is the maximum number of downlink interfaces available in an aggregation switch and $N_{\mathrm{ul}}=\left\lceil\frac{A g_{\mathrm{tot}}}{U_{\max }}\right]$ is the number of uplink interfaces number of ports used by the switch), where $A g_{\text {tot }}$ is the total traffic aggregated at all switches and $U_{\max }$ is the maximum rate supported by each uplink interface.In addition, the power consumed by each access switch is

$$
P_{\mathrm{s}}=\delta P_{\mathrm{s}, \max }+(1-\delta) \frac{A g_{\mathrm{switch}}}{A g_{\max }} P_{\mathrm{s}, \max },
$$

where $\delta \in[0,1]$ is a weighting parameter, $P_{\mathrm{s}, \max }$ is the maximum power consumed by the switch, $A g_{\text {switch }}$ is the traffic traversing the switch, and $A g_{\max }$ is the maximum traffic supported by the switch.

\section{B. Area Energy Efficiency}

In order to compare networks with different cell sizes, we define the area power consumption in $\mathrm{W} / \mathrm{km}^{2}$ as [12]

$$
\Omega=\frac{P_{\text {net }}}{A},
$$

\footnotetext{
${ }^{2}$ Let us remark that $P_{\mathrm{bh}}=0$ in [3], while $P_{1}=0$ in [13].
} 
while we also assume that the cells may have different area throughput targets, which can be written as [13]

$$
\tau_{A}=\frac{C_{\text {net }}}{A},
$$

where $C_{\text {net }}$ is the total network capacity, which is different depending on the employed MIMO scheme, as will be detailed in Section III. Finally, to reflect the ratio between the overall network capacity and the energy consumption, we adopt an area energy efficiency metric, in bits $/ \mathrm{J} / \mathrm{km}^{2}$, given by [18]

$$
\eta_{A}=\frac{\tau_{A}}{P_{\text {net }}} .
$$

\section{MIMO TRANSMISSION SCHEMES}

\section{A. Spatial Multiplexing (SM)}

In order to exploit the multiplexing gains provided by multiple antennas, SM transmits $m=\min \left\{m_{\mathrm{t}}, m_{\mathrm{r}}\right\}$ independent and separate encoded data streams, one by each transmit antenna ${ }^{3}$. Then, the average SNR per receive antenna is [3]

$$
\bar{\gamma}_{\mathrm{SM}}=\frac{\bar{\gamma}}{m}
$$

while the capacity of the SM scheme is [3], [16]

$$
C_{\mathrm{net}}^{(\mathrm{SM})}=N_{\mathrm{BS}} W \log _{2}\left[\operatorname{det}\left(\mathbf{I}_{\mathrm{m}}+\frac{\gamma_{\mathrm{SM}} \boldsymbol{\Xi}}{1+\gamma_{\mathrm{SM}} \zeta^{-1} \boldsymbol{\Xi}}\right)\right],
$$

where $\mathbf{I}_{\mathrm{m}}$ is an $m \times m$ identity matrix and $\boldsymbol{\Xi}=\mathbf{H H}^{\dagger}$ if $m_{\mathrm{t}} \geq$ $m_{\mathrm{r}}$, or $\boldsymbol{\Xi}=\mathbf{H}^{\dagger} \mathbf{H}$ otherwise, with $\mathbf{H}^{\dagger}$ being the conjugate transpose of $\mathbf{H}$.

\section{B. Transmit Antenna Selection (TAS)}

When TAS is employed, we assume that only $\widehat{m}_{\mathrm{t}}=1$ antenna is selected from the set of $m_{\mathrm{t}}$ transmit antennas, which saves power since only one RF chain remains active. Assuming maximum ratio combining (MRC) at the receiver side, the average SNR of TAS is [17]

$$
\gamma_{\mathrm{TAS}}=\bar{\gamma} \max _{i} \sum_{j=1}^{m_{\mathrm{r}}}\left|h_{i, j}\right|^{2}
$$

where the maximum over $i$ represents that only the best antenna of the transmitter is chosen, while the sum comes from the MRC at the receiver.

Finally the capacity of TAS, $C_{\text {net }}^{(\mathrm{TAS})}$, is given by

$$
C_{\mathrm{net}}^{(\mathrm{TAS})}=N_{\mathrm{BS}} W \log _{2}\left(1+\frac{\gamma_{\mathrm{TAS}}}{1+\gamma_{\mathrm{TAS}} \zeta^{-1}}\right) .
$$

Other MIMO schemes could also be considered, as e.g. Space-Time Codes or Maximal Ratio Transmission (MRT). However, these methods are outperformed by SM or TAS in terms of energy efficiency [3]. Moreover, we restrict our investigation to techniques available in current LTE deployments, especially for small BSs, so that we leave other approaches such as Massive MIMO [19] for future investigations.

\footnotetext{
${ }^{3}$ In SM, we consider that all transmit antennas are active $\left(\widehat{m}_{\mathrm{t}}=m_{\mathrm{t}}\right)$.
}

TABLE I

SYSTEM PARAMETERS

\begin{tabular}{c|l|c}
\hline Parameter & Description & Value \\
\hline$A$ & Coverage area & $41 \mathrm{~km}^{2}$ \\
$G$ & Antenna gain & $10 \mathrm{dBi}$ \\
$L$ & Link margin & $10 \mathrm{~dB}$ \\
$\alpha$ & Path-loss exponent & 3.5 \\
$P_{\min }$ & Minimum required power at cell edge & $-95 \mathrm{~dB}$ \\
$f$ & Carrier frequency & $2.5 \mathrm{GHz}$ \\
$W$ & Bandwidth & $5 \mathrm{MHz}$ \\
$N_{0}$ & Noise psd/Hz & $-174 \mathrm{dBm}$ \\
$m_{\mathrm{t}} / m_{\mathrm{r}}$ & Number of transmit/receive antennas & $\{2,4,8\}$ \\
$P_{\max }$ & Maximum transmit power & $6.31 \mathrm{~W}$ \\
$P_{0}$ & Constant for power consumption & 3.14 \\
$P_{1}$ & Power consumption dependent on $\widehat{m}_{\mathrm{t}}$ & $35 \mathrm{~W}$ \\
$P_{2}$ & Power consumption not dependent on $\widehat{m}_{\mathrm{t}}$ & $34 \mathrm{~W}$ \\
$U_{\max }$ & Maximum rate at each uplink interface & $10 \mathrm{Gbps}$ \\
$\delta$ & Weighting parameter & 0.9 \\
$\max _{\mathrm{dl}}$ & Maximum number of downlink interfaces & 24 \\
$A g_{\max }$ & Maximum traffic per switch & $24 \mathrm{Gbps}$ \\
$P_{\mathrm{s}, \max }$ & Maximum power consumed by the switch & $300 \mathrm{~W}$ \\
$P_{\mathrm{ul}}$ & Power consumed by uplink interfaces & $2 \mathrm{~W}$ \\
$P_{\mathrm{dl}}$ & Power consumed by downlink interfaces & $1 \mathrm{~W}$ \\
\hline \multicolumn{2}{|}{}
\end{tabular}

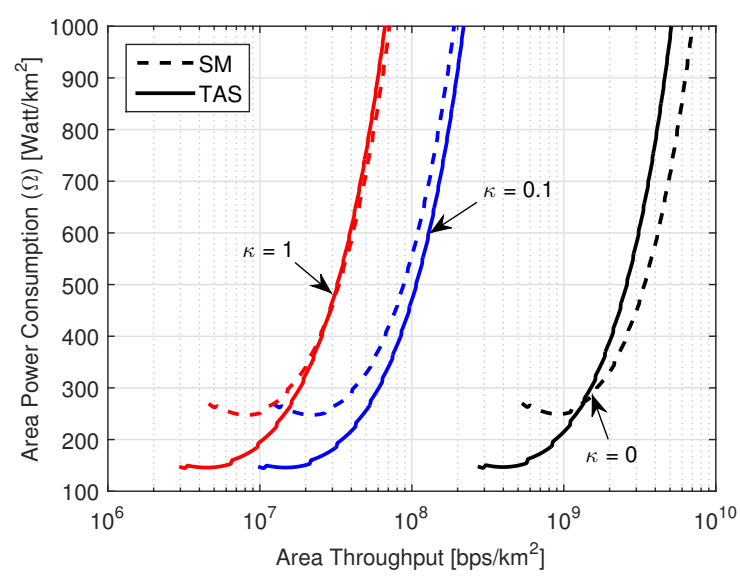

Fig. 1. Area power consumption $(\Omega)$ as a function of the area throughput $\left(\tau_{\mathrm{A}}\right)$, varying $N_{\mathrm{BS}}$, with $m_{\mathrm{t}}=m_{\mathrm{r}}=2$.

\section{NUMERICAL RESUlTS}

The simulation parameters are shown in Table I, according to [12], with the constants regarding small BS power consumption based on [3], [10] and with the power consumption parameters associated with the backhaul following [13].

Let us first analyze the area power consumption $(\Omega)$ as a function of the area throughput $\left(\tau_{\mathrm{A}}\right)$. For each scenario, there is a minimum $N_{\mathrm{BS}}$ required to cover the area $A$, which is obtained respecting the maximum transmit power $P_{\max }$ for each BS, while guaranteeing a minimum received power $\left(P_{\min }\right)$ for the UEs at the cell edge. Moreover, we also consider that a maximum of $N_{\mathrm{BS}}=500$ can be deployed. Fig. 1 plots $\Omega$ as a function of $\tau_{\mathrm{A}}$ in the case that only small BSs are employed. From the figure, we can notice that TAS minimizes the area power consumption, regardless of $\kappa$. However, if the throughput is required to be increased, different trade-offs are observed. For instance, when there is no interference at the cell edge $(\kappa=0)$, TAS is more energy-efficient than SM for 


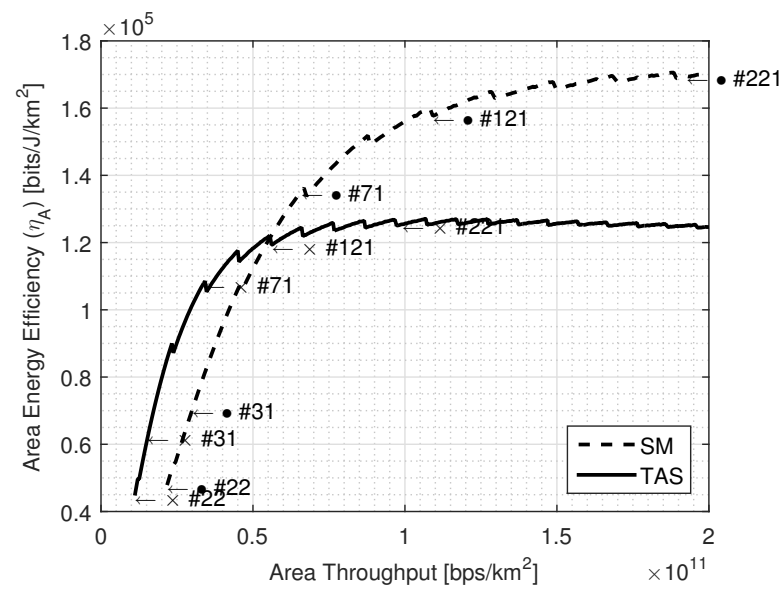

Fig. 2. Area energy efficiency $\left(\eta_{\mathrm{A}}\right)$ as a function of the area throughput $\left(\tau_{\mathrm{A}}\right)$ for small BSs with $m_{\mathrm{t}}=m_{\mathrm{r}}=2$ and $\kappa=0$. The arrow " $\leftarrow \bullet \#$ " indicates the $N_{\mathrm{BS}}$ employed by SM and " $\leftarrow \times \#$ " the $N_{\mathrm{BS}}$ employed by TAS.

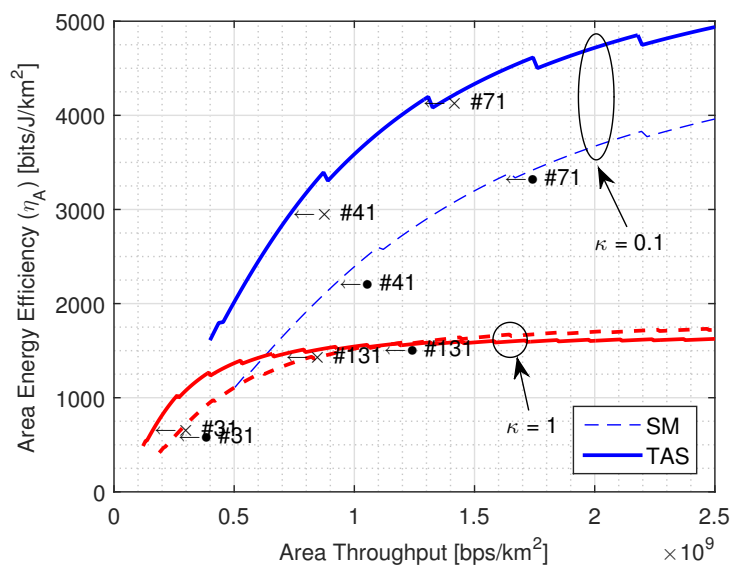

Fig. 3. Area energy efficiency $\left(\eta_{\mathrm{A}}\right)$ as a function of $\tau_{\mathrm{A}}$ for small BSs with $m_{\mathrm{t}}=m_{\mathrm{r}}=2$ and $\kappa=\{0.1,1\}$.

lower $\tau_{\mathrm{A}}$, but when $\tau_{\mathrm{A}}$ increases, TAS is slightly outperformed by SM due to the multiplexing gains that provide the required system capacity. In addition, when interference is not fully canceled and $\kappa$ increases, TAS performs better since it also generates less interference in neighbor cells. Finally, TAS is only outperformed when the required $\tau_{\mathrm{A}}$ is very high (higher than $280 \mathrm{Mbps} / \mathrm{km}^{2}$ ), which is observed when $\kappa=1$.

Moreover, Fig. 1 also shows an interesting behavior caused by the backhaul power consumption. According to (7), when a new switch must be turned on to support the traffic demand through the backhaul, $90 \%$ of $P_{\mathrm{s}, \max }$ is consumed (due to the term $\delta$ in Table I), which is higher than the power consumption of the network $\left(P_{\text {net }}\right)$ in the case of small BS. Thus, the curves exhibit a saw shape, indicating when a new switch starts.

Figures 2 and 3 show the area energy efficiency $\left(\eta_{\mathrm{A}}\right)$ as a function of $\tau_{\mathrm{A}}$, with $m_{\mathrm{t}}=m_{\mathrm{r}}=2$. In Fig. 2, $\eta_{\mathrm{A}}$ is evaluated in a scenario where all the interference is considered to be fully canceled $(\kappa=0)$. As we can observe, in the region where $\tau_{\mathrm{A}}<5.5 \times 10^{10} \mathrm{bps} / \mathrm{km}^{2}$ TAS has higher $\eta_{\mathrm{A}}$ for a given area

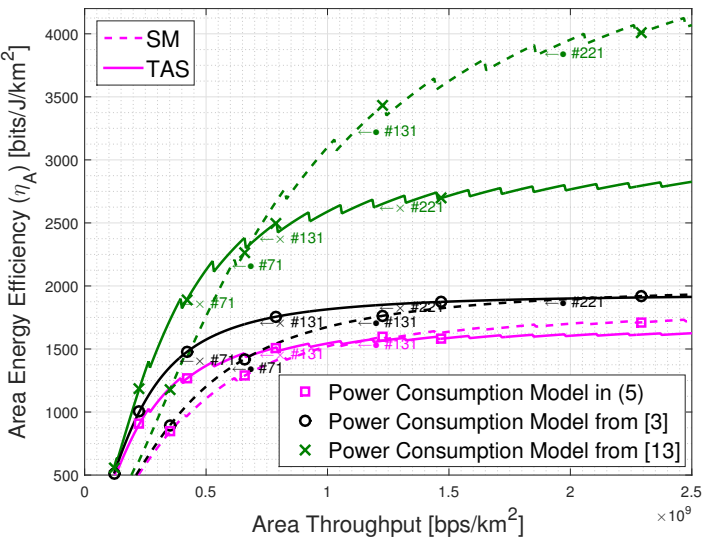

Fig. 4. Area energy efficiency $\left(\eta_{\mathrm{A}}\right)$ as a function of the area throughput $\left(\tau_{\mathrm{A}}\right)$ for small BSs with $m_{\mathrm{t}}=m_{\mathrm{r}}=2$ and $\kappa=1$. The PCM in (5) is compared with those of [3] and [13].

throughput, which is due to the energy saved by employing only one antenna for transmitting. However, SM performs better beyond that point since the multiplexing gain provided by this technique allows to use lower transmit power given the required area throughput. In addition, " $\leftarrow \bullet \#$ " indicates the $N_{\text {BS }}$ employed by SM and " $\leftarrow \times \#$ " the $N_{\text {BS }}$ employed by TAS. Notice that even when TAS is more energy efficient than SM, it requires a higher number of deployed small BSs.

Next, Fig. 3 presents the same analysis as in Fig. 2, but considering that $\kappa=0.1$ (interference is not fully canceled) and $\kappa=1$ (no interference cancellation at all). As we can observe, this analysis corroborates with the results of Fig. 1, so that TAS achieves the best performance when $\kappa=0.1$. In this case when a fixed $N_{\mathrm{BS}}$ is chosen TAS has more $\eta_{\mathrm{A}}$ and SM has higher $\tau_{\mathrm{A}}$. Moreover, notice that $\eta_{\mathrm{A}}$ is an increasing function of $N_{\mathrm{BS}}$ when $\kappa \neq 0$, so that the area energy efficiency increases with the number of base stations.

The effect of different PCMs is illustrated in Fig. 4, which, besides (5), also considers [3] which does not include the backhaul power consumption (i.e. $P_{\mathrm{bh}}=0$ ), and [13] which does not include the fraction that scales with $\widehat{m}_{\mathrm{t}}\left(\right.$ i.e. $\left.P_{1}=0\right)$. As we can observe from the curves, the intersection between TAS and SM considerably changes depending on the considered PCM. For instance, if we consider the curves when the optimal $N_{\mathrm{BS}}=131$, the PCM in [13] indicates SM as the most energy efficient scheme, with $\eta_{A} \approx 3500 \mathrm{bits} / \mathrm{J} / \mathrm{km}^{2}$, while the PCM in [3] indicates TAS, with $\eta_{A} \approx 1750 \mathrm{bits} / \mathrm{J} / \mathrm{km}^{2}$, whereas the more elaborate PCM in (5) leads to the intersection between TAS and SM, with $\eta_{A} \approx 1500 \mathrm{bits} / \mathrm{J} / \mathrm{km}^{2}$.

Figures $5 \mathrm{a}$ and $5 \mathrm{~b}$ evaluate $\eta_{\mathrm{A}}$ as a function of the number of antennas, considering that $m_{\mathrm{t}}=m_{\mathrm{r}} \in\{2,4,8\}$, and with a target network capacity of $C_{\text {net }}=1 \times 10^{11}$ bits $/ \mathrm{s}$ in Fig. 5a and $C_{\text {net }}=7 \times 10^{9}$ bits/s in Fig. 5b. As we can observe, SM performs better when the demanded network capacity is higher (Fig. 5a) and with smaller number of antennas. On the other hand, TAS outperforms the other schemes when the number of antennas is higher (with $m_{\mathrm{t}}=m_{\mathrm{r}}=8$ in Fig. 5a) or with 


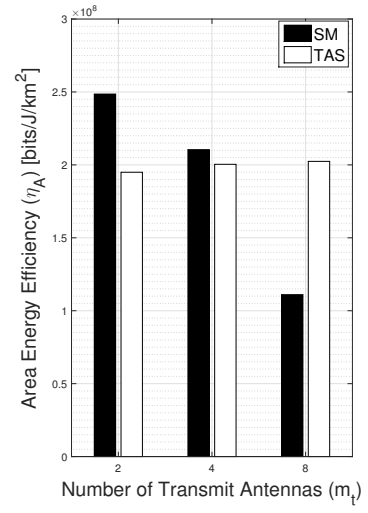

(a) $C_{\text {net }}=1 \times 10^{11} \mathrm{bits} / \mathrm{s}$

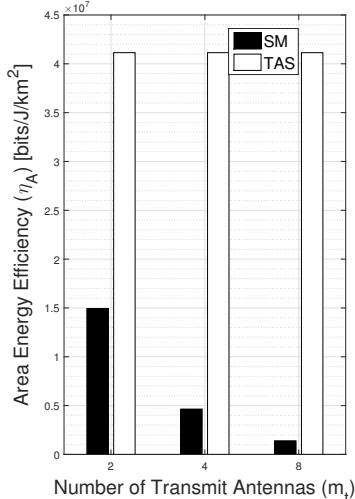

(b) $C_{\text {net }}=7 \times 10^{9}$ bits $/ \mathrm{s}$
Fig. 5. Area energy efficiency $\left(\eta_{\mathrm{A}}\right)$ as a function of the number of antennas $\left(m_{t}=m_{r}\right)$ with $\kappa=0$ and different $C_{\text {net }}$ targets.

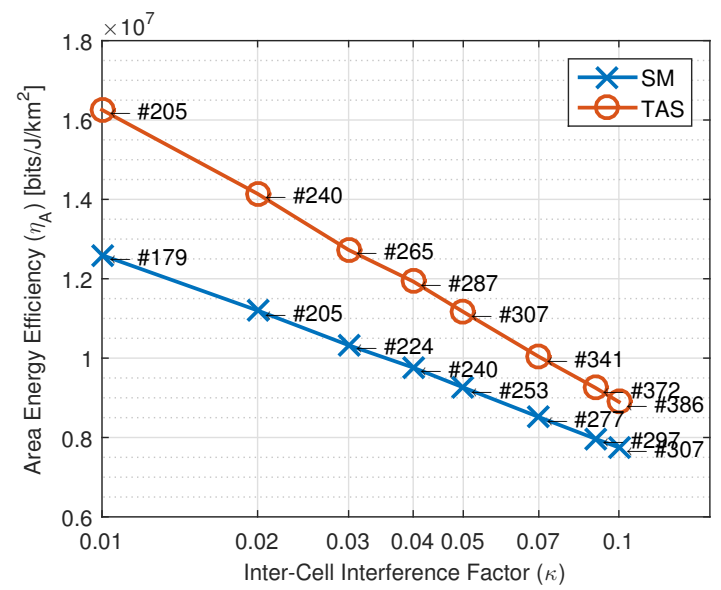

Fig. 6. Area energy efficiency $\left(\eta_{\mathrm{A}}\right)$ as a function of $\kappa$ for $m_{t}=m_{r}=2$, and with a capacity target of $7 \times 10^{9}$ bits/s.

less demand for network capacity (Fig. 5b). This behavior is explained by the lower energy consumption of TAS compared to SM, which increases its energy efficiency unless the demand for traffic is too high, so that SM performs better.

Finally, Fig. 6 evaluates the area power consumption as a function of $\kappa$, with $m_{\mathrm{t}}=m_{\mathrm{r}}=2$ and a target network capacity of $C_{\text {net }}=7 \times 10^{9}$ bits/s. Consistent with Fig. 5b, TAS achieves the highest area energy efficiency in this scenario. However, it is interesting to notice that this increased performance comes at the cost of employing more small BSs than SM to supply the same target network capacity. For each point in the curve, SM always requires less BSs than TAS, but the AEE is lower.

\section{Final Comments}

In this paper we evaluated a cellular network employing two different multiple antenna techniques: SM and TAS. The goal was to optimize the AEE by calculating the optimal number of BSs given some requirements, such as demanded network capacity, amount of interference and employed MIMO scheme. Our results show that TAS achieves the higher AEE when the demand for system capacity is lower, while SM becomes more energy efficient when the demanded capacity is higher. Additionally, when the capacity demand and the area to be covered are fixed, we also show that although achieving the highest AEE, TAS also demands more small BSs than SM. Finally, the system performance in terms of AEE is shown to be strongly dependent on the amount of interference, which in turn depends on the employed interference-mitigation scheme, and on the employed PCM, if the backhaul or the fraction that scales with the number of antennas are considered or not.

\section{REFERENCES}

[1] "Cisco visual networking index: Global mobile data traffic forecast update, 2015-2020," White Paper, Cisco, Feb. 2016.

[2] "Ericsson mobility report: On the pulse of the networked society," White Paper, Ericsson, Jun. 2016.

[3] O. K. Rayel, G. Brante, J. L. Rebelatto, R. D. Souza, and M. A. Imran, "Energy efficiency-spectral efficiency trade-off of transmit antenna selection," IEEE Trans. Commun., vol. 62, no. 12, pp. 4293-4303, Dec 2014.

[4] L. Liu, R. Chen, S. Geirhofer, K. Sayana, Z. Shi, and Y. Zhou, "Downlink MIMO in LTE-advanced: SU-MIMO vs. MU-MIMO," IEEE Commun. Mag., vol. 50, no. 2, pp. 140-147, Feb 2012.

[5] G. Auer, V. Giannini, C. Desset, I. Godor, P. Skillermark, M. Olsson, M. A. Imran, D. Sabella, M. J. Gonzalez, O. Blume, and A. Fehske, "How much energy is needed to run a wireless network?" IEEE Wireless Commun., vol. 18, no. 5, pp. 40-49, Oct 2011.

[6] H. Li, L. Song, and M. Debbah, "Energy Efficiency of Large-Scale Multiple Antenna Systems with Transmit Antenna Selection," IEEE Trans. Commun., vol. 62, no. 2, pp. 638-647, Feb 2014.

[7] N. B. Mehta, S. Kashyap, and A. F. Molisch, "Antenna selection in LTE: from motivation to specification," IEEE Commun. Mag., vol. 50, no. 10, pp. $144-150$, Oct 2012.

[8] A. F. Molisch, "MIMO systems with antenna selection - an overview," in Radio and Wireless Conference (RAWCON), Aug 2003, pp. 167-170.

[9] G. Auer, V. Giannini, I. Godor, P. Skillermark, M. Olsson, M. A. Imran, D. Sabella, M. J. Gonzalez, C. Desset, and O. Blume, "Cellular energy efficiency evaluation framework," in Vehicular Technology Conference (VTC Spring), May 2011, pp. 1-6.

[10] F. Heliot, M. A. Imran, and R. Tafazolli, "On the energy efficiencyspectral efficiency trade-off over the MIMO Rayleigh fading channel," IEEE Trans. Commun., vol. 60, no. 5, pp. 1345-1356, May 2012.

[11] F. Richter, A. J. Fehske, P. Marsch, and G. P. Fettweis, "Traffic demand and energy efficiency in heterogeneous cellular mobile radio networks," in Vehicular Technology Conference (VTC-Spring), May 2010, pp. 1-6.

[12] S. Tombaz, K. W. Sung, and J. Zander, "On metrics and models for energy-efficient design of wireless access networks," IEEE Wireless Commun. Lett., vol. 3, no. 6, pp. 649-652, Dec 2014.

[13] S. Tombaz, P. Monti, K. Wang, A. Vastberg, M. Forzati, and J. Zander, "Impact of backhauling power consumption on the deployment of heterogeneous mobile networks," in Global Telecommunications Conference (GLOBECOM), Dec 2011, pp. 1-5.

[14] M. Boujelben, S. B. Rejeb, and S. Tabbane, "A comparative study of interference coordination schemes for wireless mobile advanced systems," in Int. Symp. Netw., Comput. Commun., Jun 2014, pp. 1-5.

[15] S. Nagata, W. Xi, X. Yun, Y. Kishiyama, and L. Chen, "Interference measurement scheme for CoMP in LTE-advanced downlink," in Vehicular Technology Conference (VTC Spring), June 2013, pp. 1-6.

[16] M. H. A. Khan, J.-G. Chung, and M. H. Lee, "Downlink performance of cell edge using cooperative BS for multicell cellular network," EURASIP J. Wirel. Commun. Netw., vol. 2016, no. 1, p. 56, 2016.

[17] A. Goldsmith, Wireless Communications. New York, NY, USA: Cambridge University Press, 2005.

[18] A. A. A. Suhail Najm Shahab and A. R. Zainun, "Assessment of Area Energy Efficiency of LTE Macro Base Stations in Different Environments," J. Telecommun. and Inf. Technol., no. 1, pp. 59-66, 2015.

[19] E. Bjornson, L. Sanguinetti, J. Hoydis, and M. Debbah, "Optimal design of energy-efficient multi-user MIMO systems: Is massive MIMO the answer?" IEEE Trans. Wireless Commun., vol. 14, no. 6, pp. 3059-3075, Jun. 2015. 